\title{
Sinapic Acid Attenuated Cardiac Remodeling After Myocardial Infarction by Promoting Macrophage M2 Polarization Through PPARy Pathway
}

\section{Mei Yang}

Renmin Hospital of Wuhan University: Wuhan University Renmin Hospital https://orcid.org/0000-00020376-6592

\section{Jun Xiong}

Renmin Hospital of Wuhan University: Wuhan University Renmin Hospital

\section{Qiang Zou}

Renmin Hospital of Wuhan University: Wuhan University Renmin Hospital

\section{Xi Wang}

Renmin Hospital of Wuhan University: Wuhan University Renmin Hospital

\section{$\mathrm{Ke} \mathrm{Hu}$}

Renmin Hospital of Wuhan University: Wuhan University Renmin Hospital

Qingyan Zhao ( $\nabla$ ruyan71@163.com )

Renmin Hospital of Wuhan University: Wuhan University Renmin Hospital

\section{Research Article}

Keywords: Sinapic acid, myocardial infarction, macrophage, interstitial fibrosis, PPARY

Posted Date: January 27th, 2022

DOI: https://doi.org/10.21203/rs.3.rs-1240336/v1

License: (c) (i) This work is licensed under a Creative Commons Attribution 4.0 International License. Read Full License 


\section{Abstract}

Background Macrophage polarization is one of the important regulatory mechanisms of ventricular remodeling. Studies have shown sinapic acid (SA) exerts an anti-inflammatory role. However, the effect of SA on macrophage is still unclear. The purpose of the study was to investigate the role of SA in macrophage polarization and ventricular remodeling after myocardial infarction (MI).

Methods and results MI model was established by ligating left coronary artery. The rats with MI were treated with SA for 1 week or 4 weeks after MI. The effect of SA on bone marrow-derived macrophages (BMDMs) was also observed in vitro. Cardiac systolic dysfunction was significantly improved after SA treatment. SA reduced MCP-1 and CCR2 expressions and macrophage infiltration. SA decreased inflammatory factors TNF-a, IL-1 $\alpha$ and IL-1 $\beta$ levels and increased M2 macrophage marker CD206, Arg-1 and IL-10 levels at 1 week after MI. Myocardial interstitial fibrosis and MMP-2 and MMP-9 levels were declined, and sympathetic nerve marker TH and nerve sprouting marker GAP43 were suppressed after SA treatment at 4 weeks after MI. PPARy level was notablely upregulated after SA treatment. In vitro, SA also increased expression of PPARy mRNA in BMDMs and IL-4 induced BMDMs in a concentration-dependent manner. SA enhanced Arg1 and IL-10 expressions in BMDMs and the PPARY antagonist GW9662 attenuated M2 macrophage markers expressions.

Conclusions Our results demonstrated that SA attenuated structural and neural remodeling by promoting macrophage M2 polarization via PPARy activation after MI.

\section{Introduction}

Monocytes were recruited to the necrosis and ischemia myocardium and then become plastic macrophages after myocardial infarction (MI). A few days in the early stage of MI polarized M1 macrophages secrete pro-inflammatory mediators to activate an intense inflammatory cascade response, and polarized M2 macrophages persist for weeks to participate in fibrosis and remodeling [1]. Interstitial fibrosis after $\mathrm{Ml}$ is the main cause of heart failure and sympathetic neural remodeling contributes to ventricular arrhythmia that is closely associated with sudden cardiac death (SCD) [2, 3]. Studies have shown that modulation of macrophage polarization is a potential therapeutic target for cardiac remodeling after $\mathrm{MI}[4]$.

Sinapic acid (SA) is a hydroxycinnamic acid derivative that has a variety of biological effects, such as antioxidant, anti-inflammatory and anticancer activities. Studies have shown that SA plays an antiinflammatory role in renal and hepatic injury $[5,6]$. Recently, one study found that SA could ameliorate acute DOX-induced cardiotoxicity by inhibiting inflammation [7]. Furthermore, SA protected hypertension by modulating reactive oxygen species and inhibiting fibrosis $[8,9]$. SA also has antilipidemic and antioxidant effects in the early stage of myocardial damage $[10,11]$. However, the effects of SA on macrophage polarization and cardiac remodeling after MI have not been investigated. 
Ligand-dependent nuclear receptor peroxisome proliferator-activated receptor Y (PPARY) can regulate transcription and expression of target genes. PPARy can regulate phenotype of macrophages and participate in M2 macrophage polarization $[12,13]$. Studies reported that PPARY is expressed in macrophages and PPARy ligands can induce expression of M2 macrophage markers in vitro [14]. One recent study found that SA attenuates cisplatin-induced nephrotoxicity by PPARy activation [15]. This study intended to investigate the effect and underlying mechanism of SA on macrophage polarization and structural and neural remodeling after $\mathrm{MI}$.

\section{Materials And Methods}

\section{Animals and experimental group}

All animal procedures were performed in agreement with the Animal Care and Use Committee of Renmin Hospital of Wuhan University (Wuhan, China) and complied with the guidelines of the National Institutes of Health for the care and use of laboratory animals. SD rats (male, 6-8 weeks) were randomly divided into the sham group $(n=10)$, MI group $(n=10)$, and MI+SA group $(n=10)$. In the Ml+SA group, rats were treated with SA (Med Chem Express, USA) $(20 \mathrm{mg} / \mathrm{kg} / \mathrm{d})$ by intragastric administration $24 \mathrm{~h}$ after acute $\mathrm{MI}$ for 1 week or 4 weeks. SA was dissolved in corn oil $(5 \mathrm{mg} / \mathrm{mL})$. The rats in the MI and sham groups were administered corn oil by intragastric administration.

\section{Acute MI model establishment}

Rats were anaesthetized with an intraperitoneal injection of sodium pentobarbital ( $50 \mathrm{mg} / \mathrm{kg})$. The rats then underwent endotracheal intubation and surface electrocardiography. A thoracotomy was performed and the heart was exposed. Left anterior descending (LAD) coronary artery ligation was performed by a 60 noninvasive suture according to our previous method [16]. In the sham group, the suture was only crossed under the LAD coronary artery without ligation.

\section{Cell culture and treatment}

Bone marrow was isolated from femurs and tibias of rats according to our previous method [17]. Red blood cell lysate was added for 5 min and then washed with PBS. Cells were cultured in Dulbecco's modified Eagle's medium with $10 \%$ fetal bovine serum and $30 \mu \mathrm{g} / \mathrm{L}$ macrophage colony-stimulating factor. Following $48 \mathrm{~h}$ of culture in an incubator at $37^{\circ} \mathrm{C}$ with a $5 \% \mathrm{CO}_{2}$ atmosphere, the medium was removed and replaced with fresh medium every 3 days. BMDMs were randomly divided into six groups to observe the effect of SA on M2 polarization: a control group, IL-4 group, SA group, IL-4+SA group, SA+GW9662 group, and IL-4+SA+GW9662 group. BMDMs in the LPS group and IL-4 group were treated with LPS $(100 \mathrm{ng} / \mathrm{mL})$ or IL-4 $(10 \mathrm{ng} / \mathrm{mL})$ for $24 \mathrm{~h}$. SA (100 or $200 \mu \mathrm{mol} / \mathrm{L})$ were simultaneously added for $24 \mathrm{~h}$. In SA+GW9662 and IL-4+SA+GW9662 group, BMDMs were pre-treated with PPARY antagonist GW9662 (5 $\mu \mathrm{mol} / \mathrm{L}))(\mathrm{HY}-16578$, Med Chem Express, USA).

\section{Echocardiography}


Echocardiography was used to evaluate left ventricular (LV) systolic function after 1 week and 4 weeks. Echocardiography was recorded at the papillary muscle level in the LV short axis. The transducer frequency was $10 \mathrm{MHz}$. The parameters included LV end-diastolic dimension (LVEDD) and LV ejection fraction (LVEF).

\section{Western blot analysis}

Total protein was extracted from myocardial tissue in the peripheral zone of MI and BMDMs, and the BCA method was used to determine the protein concentration. Sodium dodecyl sulfate-polyacrylamide gel electrophoresis was performed, and the proteins were transferred to a polyvinylidene difluoride membrane. The membrane was incubated with primary antibodies against MCP-1 (Bioss, bs-34021R, 1:500), CCR2 (Biorbyt, orb378630, 1:1000), Arg-1 (Santa, sc-271430, 1:500), MMP-2 (Abcam, ab92536, 1:1000), MMP-9 (Abcam, ab76003, 1:1000), PPARy (Abcam, ab209350, 1:1000) overnight at $4^{\circ} \mathrm{C}$ and then incubated with secondary antibody HRP-goat anti-rabbit (ASPEN, AS1107) at $37^{\circ} \mathrm{C}$ for $45 \mathrm{~min}$; then, enhanced chemiluminescence detection was performed. Optical density was detected by the AlphaEaseFC software system.

\section{Reverse transcription-quantitative polymerase chain reaction (RT-qPCR)}

Total RNA was extracted from the peripheral area of infarction by using TRIzol@ reagent. The isolated RNA was converted into cDNA using the PrimeScript ${ }^{\text {TM }}$ RT reagent kit with gDNA Eraser (TaKaRa, RR047A). The primers were synthesized by Invitrogen Biotechnology (Shanghai, China) and presented in Table 1. RT-qPCR was performed using the StepOne ${ }^{\mathrm{TM}}$ Real-Time PCR system (Life Technologies, Carlsbad, CA, USA). The reactions were then conducted using SYBR® Premix Ex Taq TM II (Takara Bio, Japan, RR420A). Semilog amplification curves were analyzed using the 2- $\Delta \Delta \mathrm{Ct}$ comparative quantification method, and the expression of each gene was normalized to GAPDH. 
Table 1

PCR primers used in this study

\begin{tabular}{|c|c|}
\hline Gene & Primer \\
\hline \multirow[t]{2}{*}{ TNF-a } & Sense:5'- CACCACGCTCTTCTGTCTACTG - 3' \\
\hline & Antisense:5'- GCTACGGGCTTGTCACTCG -3' \\
\hline \multirow[t]{2}{*}{ IL-1a } & Sense:5'- GCAAAGAAATCAAGATGGCCA - 3' \\
\hline & Antisense:5'- CATGAAGTGAGCCATAGCTTGC-3' \\
\hline \multirow[t]{2}{*}{ IL-1 $\beta$} & Sense:5'- GTGGCAGCTACCTATGTCTTGC - 3' \\
\hline & Antisense:5'- CCACTTGTTGGCTTATGTTCTGT -3' \\
\hline \multirow[t]{2}{*}{ IL-10 } & Sense:5'- GCAGGACTTTAAGGGTTACTTGG - 3' \\
\hline & Antisense:5'- АTCATTCTTCACCTGCTCCACT -3' \\
\hline \multirow[t]{2}{*}{ NGF } & Sense:5'- AGAACCGTACACAGATAGCAATGTC - 3' \\
\hline & Antisense:5'- GTCCTGTTGAAGGAGATTGTACCAT -3 \\
\hline \multirow[t]{2}{*}{ PPARY } & Sense:5'- CCCTTTACCACGGTTGATTTC - 3' \\
\hline & Antisense:5'- CTTCAATCGGATGGTTCTTCG -3' \\
\hline \multirow[t]{2}{*}{ Arg-1 } & Sense:5'- AACACTCCCCTGACAACCA - 3' \\
\hline & Antisense:5'- CATCACCTTGCCAATCCC -3' \\
\hline \multirow[t]{2}{*}{ GAPDH } & Sense:5'- CGCTAACATCAAATGGGGTG -3' \\
\hline & Antisense:5'- TTGCTGACAATCTTGAGGGAG -3' \\
\hline
\end{tabular}

\section{Masson trichrome staining}

The myocardial tissue in the peripheral infarct zones was fixed with $4 \%$ paraformaldehyde and embedded in paraffin. The sections were dewaxed, dehydrated, and stained with hematoxylin. Next, the sections were stained with Ponceau and aniline blue after washing and sealed with neutral gum. Collagen volume fraction (CVF) was defined as the ratio of collagen fiber area to the view area.

\section{Immunofluorescence}

The paraffin sections were dewaxed and hydrated, and then blocked with $5 \%$ bovine serum albumin (BSA) for $20 \mathrm{~min}$. Next, the sections were incubated with the primary antibodies CD68 (Abcam, ab125212; 1:200) and CD206 (Abcam, ab64693; 1:200) overnight at $4^{\circ} \mathrm{C}$ and the secondary antibodies Cy3conjugated AffiniPure goat anti-rabbit IgG (Aspen, AS11079) at $37^{\circ} \mathrm{C}$ for 50 min. 4',6-Diamidino-2phenylindole (DAPI) was used to visualize the nuclei. The sections were observed by the ScanScope system. Four visual fields were randomly selected in every section. The CD68+ and CD206+ count was the number of CD68-positive cells and CD206-positive cells per field. 


\section{Immunohistochemistry}

The primary antibodies TH (Abcam, ab109189, 1:200) and GAP43 (Abcam, ab128005, 1:150) were added to the sections overnight at $4^{\circ} \mathrm{C}$. Then the sections were incubated with secondary antibody horseradish peroxidase-conjugated goat anti-rabbit lgG (Aspen, AS1107) at $37^{\circ} \mathrm{C}$ for $50 \mathrm{~min}$. Then, the sections were stained with diaminobenzidine solution. Four visual fields were randomly selected in every section. The mean integral optical density of TH and GAP43 was measured by Image-Pro plus software.

\section{Statistical analysis}

The data are expressed as the mean \pm SD. The statistical significance of the differences among groups was determined using one-way analysis of variance (ANOVA) with a Tukey post-hoc test. $P<0.05$ was considered to indicate a statistically significant difference.

\section{Results}

\section{SA ameliorated cardiac systolic dysfunction}

In the MI group, LVEDD increased and LVEF decreased compared with the sham group after 1 week and 4 weeks (Fig. 1a-c). After SA treatment, LVEDD was reduced and LVEF were improved compared with the MI group. SA significantly ameliorated LV dysfunction after MI (Fig. 1a-c). There was no significant difference in LVEDD and LVEF between 1 and 4 weeks after myocardial infarction in the three groups.

\section{$S A$ reduced macrophages infiltration and promoted macrophage M2 polarization}

The effect of SA on macrophage was analyzed at 1 week after MI. MCP-1/CCR2 plays an important role in migration and infiltration of macrophages after MI [18]. SA markedly reduced the increased MCP-1 and CCR2 protein levels after MI (Fig. 1d, e). The inflammatory factors TNF-a, IL-1a and IL-1 $\beta$ were declined in the Ml+SA group compared with the MI group (Fig. 1f). The effect of SA on macrophage polarization was further analyzed and our study found that SA significantly enhanced M2 marker Arg-1 and IL-10 levels (Fig. 1d-f). Immunofluorescence analysis showed that the expressions of general macrophage marker CD68 and M2 macrophage marker CD206 were both upregulated in the MI group compared with the sham group. CD 68 positive cells infiltration was greatly decreased and CD206 positive cells infiltration was greatly increased following SA treatment at 1 week after MI (Fig. 2a-c).

\section{SA attenuated myocardial fibrosis}

Myocardial fibrosis was evaluated by collagen synthesis and collagen degradation. Masson staining and quantitative analysis of CVF indicated that SA significantly attenuated interstitial fibrosis after 4 weeks of acute MI (Fig. 2d, e). MMP-2 and MMP-9 protein levels were also elevated after Ml and markedly reduced after SA treatment (Fig. 2f, g).

\section{Effect of SA on neural remodeling}


Sympathetic nerve marker TH and nerve sprouting marker GAP43 were evaluated by immunohistochemistry. There was a small amount of TH-positive and GAP43-positive nerves in the sham group (Fig. 3a). At 4 weeks after Ml, cardiac sympathetic nerve fibers and nerve sprouting were significantly increased in the peripheral area of MI (Fig. 3a-C). Compared with the MI group, the results of nerve density measurements indicated that TH-positive and GAP43-positive nerves were notably lower in the MI+SA group (Fig. 3a-C). Furthermore, SA inhibited NGF expression after MI. (Fig. 3d).

\section{Effect of SA on the expression of PPARy in vivo and effect of SA on BMDMs}

The expression of PPARy protein was upregulated at 1 week after MI. In the MI+SA group, PPARY expression significantly elevated compared with the MI group (Fig. 4a, b). To further investigate the mechanism of SA on macrophage, we observed the effect of SA on BMDMs in vitro. Our study found that SA also acticvated expression of PPARY mRNA in BMDMs and IL-4 induced BMDMs in a concentrationdependent manner (Fig. 4C). SA and IL-4 both enhanced Arg1 and IL-10 expressions, and there was no significant difference in Arg1 expression between the two groups. SA also elevated Arg1 and IL-10 levels in IL-4 induced BMDMs (Fig. 4d, e). The PPARy antagonist GW9662 attenuated the SA and IL-4+SA induced M2 macrophage markers expressions (Fig. 4d, e).

\section{Discussion}

This study explored the influence of SA on macrophage polarization and ventricular remodeling in rat with MI model. We provide evidence for the following: (1) SA increased M2 macrophages infiltration after $\mathrm{Ml}$; (2) SA attenuated myocardial interstitial fibrosis and neural remodeling after Ml; (3) In vitro study we further found that SA induced and promoted macrophage M2 polarization in BMDMs and IL-4 induced BMDMs; and (4) Activation of PPARY is a potential mechanism by which SA regulates macrophage polarization.

The plasticity of macrophages makes macrophages an important regulatory target for the treatment of myocardial infarction. After myocardial infarction, pro-inflammatory M1 macrophages aggravate inflammatory response and myocardial injury by releasing pro-inflammatory cytokines, exosomes and miRNA [19]. An excessive inflammatory response and prolonged M1 macrophage accelerates myocardial injury and adverse cardiac remodeling $[20,21]$. Previous studies have shown that SA can inhibit inflammation [5-7]. Our study found that SA can inhibit macrophage infiltration in peri-infarct area. MCP1 , a ligand of $G$ protein coupled receptor CCR2, recruits monocytes to the infarcted myocardium and increases macrophage infiltration to promote the inflammatory microenvironment [22]. The expression of MCP-1 continuously markedly up-regulated from 1 day to 4 weeks after myocardial infarction, and MCP1/CCR2 inhibition significantly ameliorates macrophage recruitment and interstitial fibrosis, and improves heart function after ischemia and reperfusion injury and MI $[22,23]$. We found that SA not only downregulated the inflammatory factors TNF- $\alpha$, IL-1 $a$ and IL-1 $\beta$ but also modulated macrophage polarization after MI. Our in vivo and in vitro experiments investigated that SA can promote the polarization of M2 macrophages. M2 macrophages infiltration begin to activate at 5 to 7 days after 
myocardial infarction [24, 25], and we observe M2 macrophages at 1 week after MI and in IL-4 induced BMDMs. IL-4 was applied to modulate macrophage polarization toward an M2 phenotype. IL-4 administration can significantly increase M2 macrophages infiltration and reduce the area of myocardial infarction and improve cardiac function in mice with myocardial infarction, and this effect depends on M2 macrophages rather than the direct effect of IL-4 [26]. M2 macrophages can secrete various antiinflammatory cytokines, such as IL-10 and IL-1RA. We speculate promoting M2 cell polarization is one of the important anti-inflammatory mechanisms of SA and SA did not affect the polarization of M1 macrophages.

A few weeks and months after $\mathrm{Ml}$, the imbalance of homeostasis and increase in extracellular matrix synthesis and deposition play an important role in structural remodeling. Previous studies have shown that SA prevents cardiac fibrosis in a hypertensive animal model [8]. Persistent inflammatory response recruits and activates myofibroblasts that synthesize extracellular matrix proteins, which are involved in myocardial remodeling. Our study demonstrated that not only cardiac fibrosis but also MMP-2 and MMP9 expression was inhibited after SA treatment. MMP-2 and MMP-9 are key regulators of LV remodeling and were upregulated both in MI and heart failure [27]. In MMP2 and MMP9 knockout mice, LV enlargement and collagen accumulation were significantly attenuated after MI [28, 29]. Clinical studies have also shown that MMP-9 is an independent risk factor for heart failure after acute MI [30]. Our study indicated that MMP2 and MMP9 is a regulatory target of anti-fibrosis effect of SA. Interstitial fibrosis gradually leads to impaired cardiac function and eventually progresses to heart failure. Our study also demonstrated that ventricle dilatation and systolic dysfunction following MI were evidently ameliorated after SA treatment.

Inflammatory response contributes to sympathetic neural remodeling that plays an important role in cardiac arrhythmias and SCD after MI. Macrophages that synthesize and express NGF around sympathetic nerves participate in sympathetic sprouting after MI [31]. Inflammatory factor TNF- $a$ and IL-1 $\beta$ also directly regulate the expression of NGF [32]. Furthermore, macrophage reduction followed by intravenous injection of clodronate inhibited sympathetic hyperinnervation after MI [33]. NGF secretion decreased significantly after M2 polarization of microglia [34]. Another study showed that atorvastatin induced M2 macrophages and attenuated sympathetic hyperinnervation in rat post myocardial infarction [35]. SA may inhibit NGF expression and regulate sympathetic remodeling and nerve sprouting by alleviating inflammatory response.

PPARy is closely associated with M2 polarization [12]. Some drugs, such as rosuvastatin and pioglitazone can improve M2 macrophage polarization by PPARy activation [36, 37]. IL-4 stimulation can induce BMDMs to M2 polarized activation and elevate PPARy expression [38]. In IL-4 stimulated PPARY null BMDMs, the expression of M2 macrophage marker Arg-1 were reduced by nearly half [13]. More importantly, there is PPAR response element (PPRE) within upstream of Arg-1, Ym-1, and Fizz-1 promoter to regulate the transcription of target genes $[12,13]$. One recent study found that SA possesses a PPARY activation role and the antioxidant stress effect of sinapic acid was abolished by the PPARY inhibitor BADGE [15]. In our study, we also found SA that activated PPARy in BMDMs in a concentration-dependent 
manner. More importantly, PPARy antagonist GW9662 attenuated the IL-4 induced M2 macrophage markers expressions after SA treatment, which indicated that PPARY is the core signaling pathway of SA in regulating macrophage polarization. However, the specific mechanism of SA-mediated PPARY activation remains to be further studied.

In conclusion, SA alleviated inflammation by promoting M2 macrophage polarization via activating PPARY pathway, and SA attenuated structural and neural remodeling by inhibiting inflammation. SA could be a therapeutic candidate for anti-inflammation and ventricular remodeling after MI.

\section{Declarations}

\section{Data Availability}

The data used to support the findings of this study are available from the corresponding authors upon request.

Funding This work was supported by the National Natural Science Foundation of China (Grant numbers 81970082 and 81970277$)$.

Conflict of interest The authors declare that there are no conflict of interest associated with the manuscript.

Author Contributions All authors contributed to the study conception and design. MY, JX and QZ performed the experiments. MY and JX were responsible for drafting manuscript. $\mathrm{KH}$ and $\mathrm{XW}$ assisted in the design of the study and performed the statistical analysis. QYZ and $\mathrm{KH}$ revised the manuscript. $\mathrm{KH}$ and QYZ confirm the authenticity of all the raw data. All authors have read and approved the final version of the manuscript.

Ethical approval All animal procedures were performed in agreement with the Animal Care and Use Committee of Renmin Hospital of Wuhan University.

Consent to participate and publish All authors reviewed and approved the final version for publication.

\section{References}

1. Kim Y, Nurakhayev S (2021) Macrophage Polarization in Cardiac Tissue Repair Following Myocardial Infarction. Int J Mol Sci 22:2715. https://doi.org/10.3390/ijms22052715

2. Li CY, Li YG (2015) Cardiac Sympathetic Nerve Sprouting and Susceptibility to Ventricular Arrhythmias after Myocardial Infarction. Cardiology research and practice 2015:698368. https://doi.org/10.1155/2015/698368

3. Chen PS, Chen LS, Cao JM, Sharifi B, Karagueuzian HS, Fishbein MC (2001) Sympathetic nerve sprouting, electrical remodeling and the mechanisms of sudden cardiac death. Cardiovascular Res 
50:409-416. https://doi.org/10.1016/s0008-6363(00)00308-4

4. Zhang Z, Tang J, Cui X, Qin B, Zhang J, Zhang L, Zhang H, Liu G, Wang W, Zhang J (2021) New Insights and Novel Therapeutic Potentials for Macrophages in Myocardial Infarction. Inflammation 44:1696-1712. https://doi.org/10.1007/s10753-021-01467-2

5. Ansari MA, Raish M, Ahmad A, Alkharfy KM, Ahmad SF, Attia SM, Alsaad AMS, Bakheet SA (2017) Sinapic acid ameliorate cadmium-induced nephrotoxicity: In vivo possible involvement of oxidative stress, apoptosis, and inflammation via NF-kB downregulation. Environ Toxicol Pharmacol 51:100107. https://doi.org/10.1016/j.etap.2017.02.014

6. Shin DS, Kim KW, Chung HY, Yoon S, Moon JO (2013) Effect of sinapic acid against carbon tetrachloride-induced acute hepatic injury in rats. Arch Pharm Res 36:626-633. https://doi.org/10.1007/s12272-013-0050-5

7. Bin Jardan YA, Ansari MA (2020) Sinapic Acid Ameliorates Oxidative Stress, Inflammation, and Apoptosis in Acute Doxorubicin-Induced Cardiotoxicity via the NF-KB-Mediated Pathway. 2020:3921796. https://doi.org/10.1155/2020/3921796

8. Silambarasan T, Manivannan J, Krishna Priya M, Suganya N, Chatterjee S, Raja B (2014) Sinapic acid prevents hypertension and cardiovascular remodeling in pharmacological model of nitric oxide inhibited rats. PLoS ONE 9:e115682. https://doi.org/10.1371/journal.pone.0115682

9. Aldubayan MA, Ahmed AS (2020) Sinapic Acid Attenuates Cardiovascular Disorders in Rats by Modulating Reactive Oxygen Species and Angiotensin Receptor Expression. 2020:1436858. https://doi.org/10.1155/2020/1436858

10. Roy SJ, Mainzen Prince PS (2013) Protective effects of sinapic acid on cardiac hypertrophy, dyslipidaemia and altered electrocardiogram in isoproterenol-induced myocardial infarcted rats. Eur J Pharmacol 699:213-218. https://doi.org/10.1016/j.ejphar.2012.11.012

11. Silambarasan T, Manivannan J, Priya MK, Suganya N, Chatterjee S, Raja B (2015) Sinapic acid protects heart against ischemia/reperfusion injury and $\mathrm{H} 9 \mathrm{c} 2$ cardiomyoblast cells against oxidative stress. Biochem Biophys Res Commun 456:853-859. https://doi.org/10.1016/j.bbrc.2014.12.022

12. Bouhlel MA, Derudas B, Rigamonti E, Dièvart R, Brozek J, Haulon S, Zawadzki C, Jude B, Torpier G, Marx N, Staels B, Chinetti-Gbaguidi G (2007) PPARgamma activation primes human monocytes into alternative M2 macrophages with anti-inflammatory properties. Cell Metabol 6:137-143. https://doi.org/10.1016/j.cmet.2007.06.010

13. Odegaard JI, Ricardo-Gonzalez RR, Goforth MH, Morel CR, Subramanian V, Mukundan L, Red Eagle A, Vats D, Brombacher F, Ferrante AW, Chawla A (2007) Macrophage-specific PPARgamma controls alternative activation and improves insulin resistance. Nature 447:1116-1120. https://doi.org/10.1038/nature05894

14. Penas F, Mirkin GA, Vera M, Cevey Á, González CD, Gómez MI, Sales ME, Goren NB (2015) Treatment in vitro with PPARa and PPARy ligands drives M1-to-M2 polarization of macrophages from T. cruziinfected mice. Biochim Biophys Acta 1852:893-904. https://doi.org/10.1016/j.bbadis.2014.12.019 
15. Singh HP, Singh TG, Singh R (2020) Sinapic acid attenuates cisplatin-induced nephrotoxicity through peroxisome proliferator-activated receptor gamma agonism in rats. Journal of pharmacy \& bioallied sciences 12:146-154. https://doi.org/10.4103/jpbs.JPBS_220_19

16. Yang M, Xiong J, Zou Q, Wang DD, Huang CX (2018) Chrysin attenuates interstitial fibrosis and improves cardiac function in a rat model of acute myocardial infarction. $J$ Mol Histol 49:555-565. https://doi.org/10.1007/s10735-018-9793-0

17. Li Y, Yang M, Zhang G, Li L, Ye B, Huang C, Tang Y (2018) Transcription factor TBX18 promotes adult rat bone mesenchymal stem cell differentiation to biological pacemaker cells. Int J Mol Med 41:845851. https://doi.org/10.3892/ijmm.2017.3259

18. Frangogiannis NG (2007) Chemokines in ischemia and reperfusion. Thromb Haemost 97:738-747

19. Liu S, Chen J, Shi J, Zhou W, Wang L, Fang W, Zhong Y, Chen X, Chen Y, Sabri A, Liu S (2020) M1-like macrophage-derived exosomes suppress angiogenesis and exacerbate cardiac dysfunction in a myocardial infarction microenvironment. Basic Res Cardiol 115:22. https://doi.org/10.1007/s00395020-0781-7

20. Liu X, Chen J, Zhang B, Liu G, Zhao H, Hu Q (2019) KDM3A inhibition modulates macrophage polarization to aggravate post-MI injuries and accelerates adverse ventricular remodeling via an IRF4 signaling pathway. Cell Signal 64:109415. https://doi.org/10.1016/j.cellsig.2019.109415

21. Troidl C, Möllmann H, Nef H, Masseli F, Voss S, Szardien S, Willmer M, Rolf A, Rixe J, Troidl K, Kostin S, Hamm C, Elsässer A (2009) Classically and alternatively activated macrophages contribute to tissue remodelling after myocardial infarction. J Cell Mol Med 13:3485-3496. https://doi.org/10.1111/j.1582-4934.2009.00707.x

22. Hayashidani S, Tsutsui H, Shiomi T, Ikeuchi M, Matsusaka H, Suematsu N, Wen J, Egashira K, Takeshita A (2003) Anti-monocyte chemoattractant protein-1 gene therapy attenuates left ventricular remodeling and failure after experimental myocardial infarction. Circulation 108:2134-2140. https://doi.org/10.1161/01.cir.0000092890.29552.22

23. Liehn EA, Piccinini AM, Koenen RR, Soehnlein O, Adage T, Fatu R, Curaj A, Popescu A, Zernecke A, Kungl AJ, Weber C (2010) A new monocyte chemotactic protein-1/chemokine CC motif ligand-2 competitor limiting neointima formation and myocardial ischemia/reperfusion injury in mice. J Am Coll Cardiol 56:1847-1857. https://doi.org/10.1016/j.jacc.2010.04.066

24. Ben-Mordechai T, Palevski D, Glucksam-Galnoy Y, Elron-Gross I, Margalit R, Leor J (2015) Targeting macrophage subsets for infarct repair. J Cardiovasc Pharmacol Therap 20:36-51. https://doi.org/10.1177/1074248414534916

25. Mouton AJ, DeLeon-Pennell KY, Rivera Gonzalez OJ, Flynn ER, Freeman TC, Saucerman JJ, Garrett MR, Ma Y, Harmancey R, Lindsey ML (2018) Mapping macrophage polarization over the myocardial infarction time continuum. Basic Res Cardiol 113:26. https://doi.org/10.1007/s00395-018-0686-X

26. Shintani Y, Ito T, Fields L, Shiraishi M, Ichihara Y, Sato N, Podaru M, Kainuma S, Tanaka H, Suzuki K (2017) IL-4 as a Repurposed Biological Drug for Myocardial Infarction through Augmentation of 
Reparative Cardiac Macrophages: Proof-of-Concept Data in Mice. Sci Rep 7:6877. https://doi.org/10.1038/s41598-017-07328-z

27. DeLeon-Pennell KY, Meschiari CA, Jung M, Lindsey ML (2017) Matrix Metalloproteinases in Myocardial Infarction and Heart Failure. Prog Mol Biol Transl Sci 147:75-100. https://doi.org/10.1016/bs.pmbts.2017.02.001

28. Ducharme A, Frantz S, Aikawa M, Rabkin E, Lindsey M, Rohde LE, Schoen FJ, Kelly RA, Werb Z, Libby P, Lee RT (2000) Targeted deletion of matrix metalloproteinase-9 attenuates left ventricular enlargement and collagen accumulation after experimental myocardial infarction. J Clin Investig 106:55-62. https://doi.org/10.1172/jci8768

29. Hayashidani S, Tsutsui H, Ikeuchi M, Shiomi T, Matsusaka H, Kubota T, Imanaka-Yoshida K, Itoh T, Takeshita A (2003) Targeted deletion of MMP-2 attenuates early LV rupture and late remodeling after experimental myocardial infarction. Am J Physiol Heart Circ Physiol 285:H1229-1235. https://doi.org/10.1152/ajpheart.00207.2003

30. Wagner DR, Delagardelle C, Ernens I, Rouy D, Vaillant M, Beissel J (2006) Matrix metalloproteinase-9 is a marker of heart failure after acute myocardial infarction. J Card Fail 12:66-72.

https://doi.org/10.1016/j.cardfail.2005.08.002

31. Hasan W, Jama A, Donohue T, Wernli G, Onyszchuk G, Al-Hafez B, Bilgen M, Smith PG (2006) Sympathetic hyperinnervation and inflammatory cell NGF synthesis following myocardial infarction in rats. Brain Res 1124:142-154. https://doi.org/10.1016/j.brainres.2006.09.054

32. Takano S, Uchida K (2016) Nerve Growth Factor Regulation by TNF-a and IL-1 $\beta$ in Synovial Macrophages and Fibroblasts in Osteoarthritic Mice. 2016:5706359.

https://doi.org/10.1155/2016/5706359

33. Wernli G, Hasan W, Bhattacherjee A, van Rooijen N, Smith PG (2009) Macrophage depletion suppresses sympathetic hyperinnervation following myocardial infarction. Basic Res Cardiol 104:681-693. https://doi.org/10.1007/s00395-009-0033-3

34. Zhou D, Ji L, Chen Y (2020) TSPO Modulates IL-4-Induced Microglia/Macrophage M2 Polarization via PPAR-y Pathway. J Mol Neurosci 70:542-549. https://doi.org/10.1007/s12031-019-01454-1

35. Yang N, Cheng W, Hu H, Xue M, Li X, Wang Y, Xuan Y, Li X, Yin J, Shi Y, Yan S (2016) Atorvastatin attenuates sympathetic hyperinnervation together with the augmentation of $\mathrm{M} 2$ macrophages in rats postmyocardial infarction. Cardiovasc Ther 34:234-244. https://doi.org/10.1111/1755-5922.12193

36. Zhang T, Shao B, Liu GA (2017) Rosuvastatin promotes the differentiation of peripheral blood monocytes into M2 macrophages in patients with atherosclerosis by activating PPAR-y. Eur Rev Med Pharmacol Sci 21:4464-4471

37. Zhang C, Zhang Y, Zhang C, Liu Y, Liu Y, Xu G (2019) Pioglitazone increases VEGFR3 expression and promotes activation of $\mathrm{M} 2$ macrophages via the peroxisome proliferator-activated receptor $\mathrm{Y}$. Mol Med Rep 19:2740-2748. https://doi.org/10.3892/mmr.2019.9945

38. Ying W, Cheruku PS, Bazer FW, Safe SH, Zhou B (2013) Investigation of macrophage polarization using bone marrow derived macrophages. Journal of visualized experiments: JoVE 76:50323. 


\section{Figures}

a

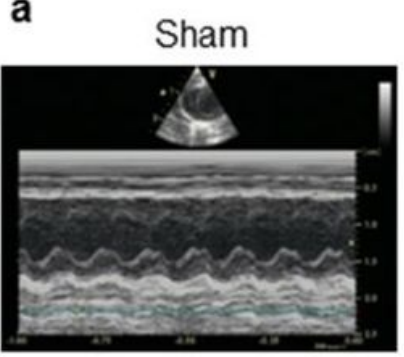

MI

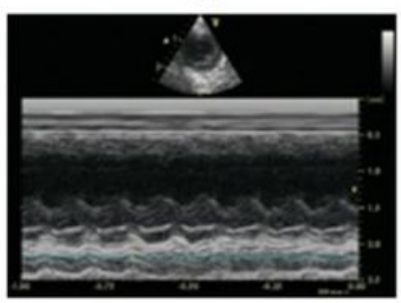

d

Sham Ml Ml+SA

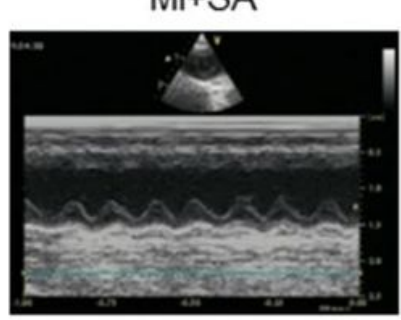

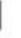
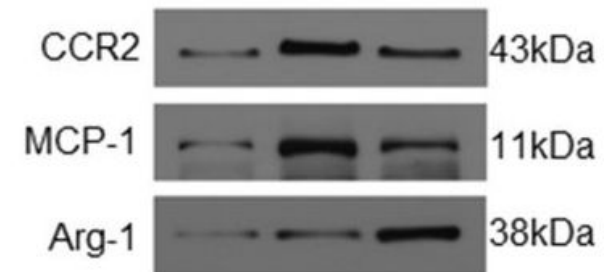

GAPDH

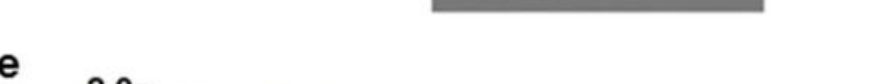

b

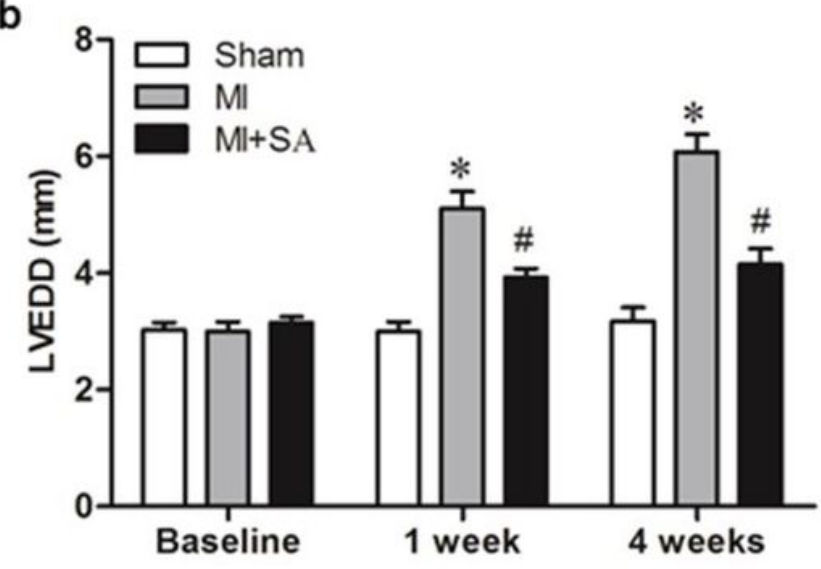

c

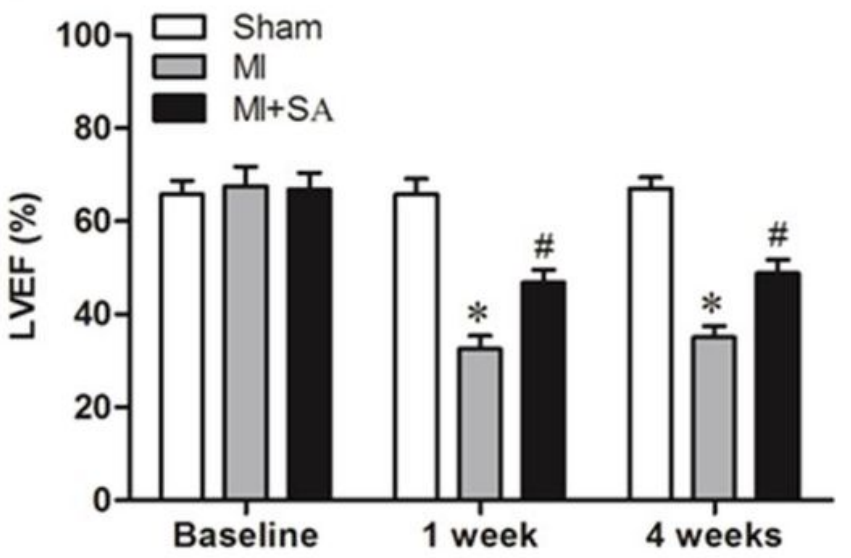

e

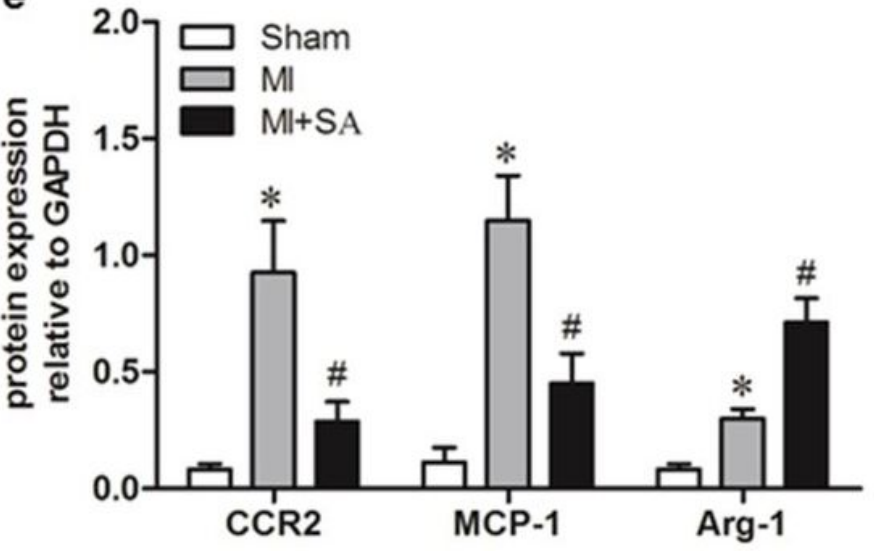

f

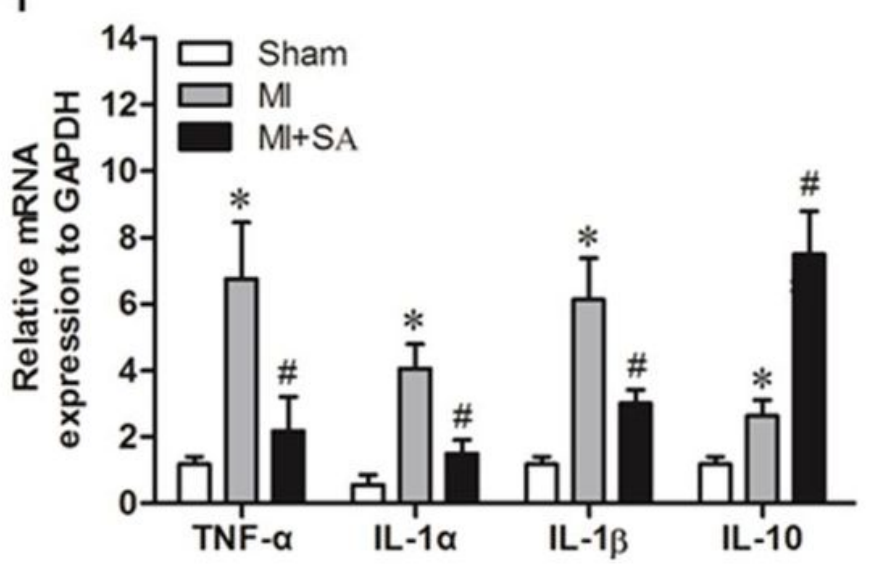

Figure 1

SA ameliorated cardiac dysfunction and modulated macrophage polarization and inflammatory factors after MI. a Echocardiographic images in the three groups at 1 week after MI. $\mathbf{b}$ and c SA decreased LVEDD and increased LVEF at 1 week and 4 weeks after MI. $\mathbf{d}$ and e MCP-1, CCR2 and M2 marker Arg-1 expressions in the three groups were detected by Western blot. $\mathbf{f}$ Levels of inflammatory factors TNF-a, IL$1 \mathrm{a}, \mathrm{IL}-1 \beta$ and IL-10 were detected by RT-PCR. ${ }^{*}$ compared with the sham group, $\mathrm{P}<0.05$, \#compared with the Ml+SA group, $\mathrm{P}<0.05$ 


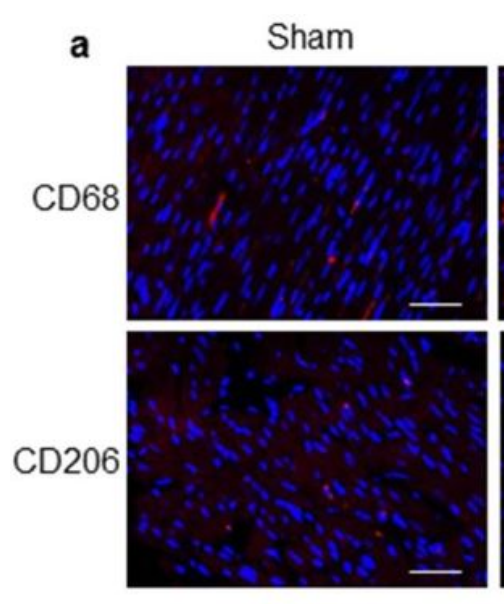

d
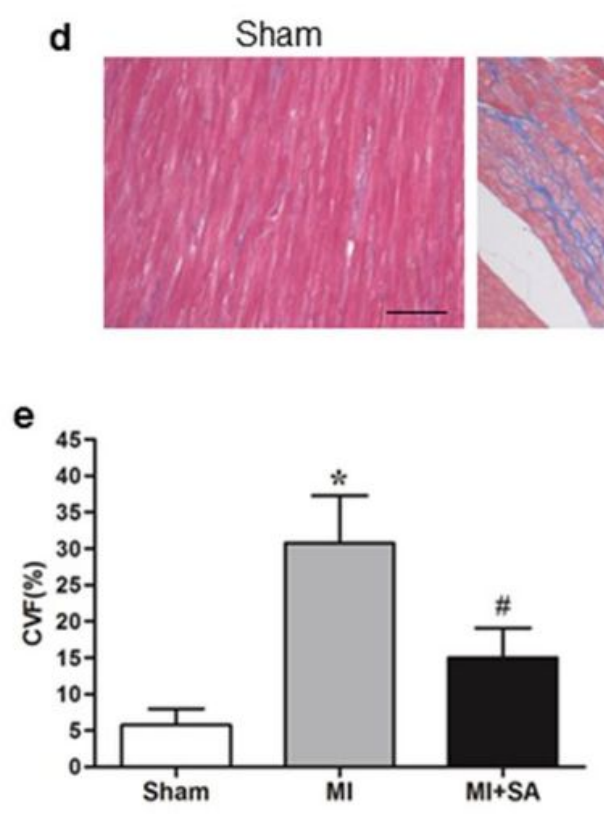

Ml
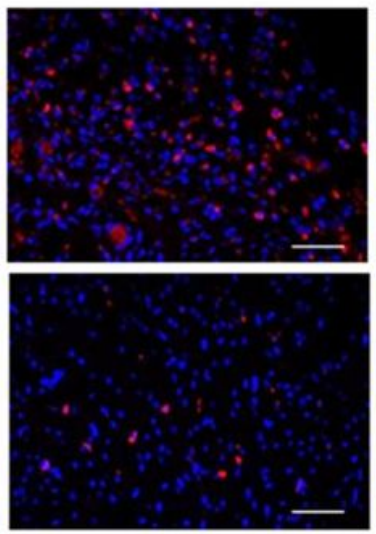

$\mathrm{Ml}$

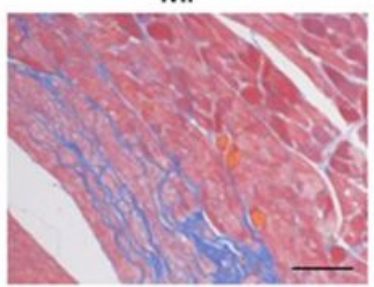

f

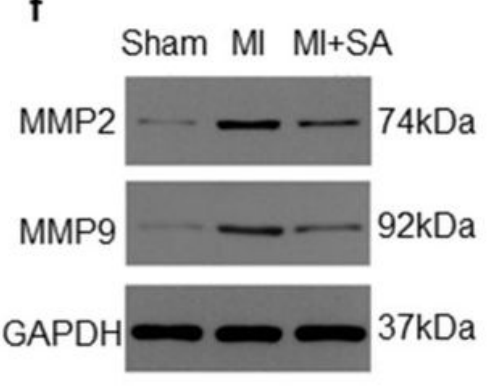

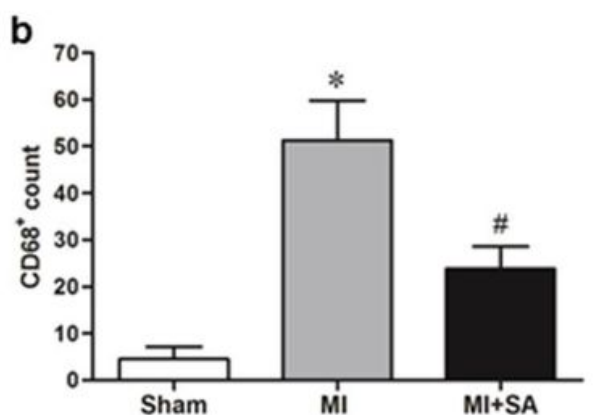

c
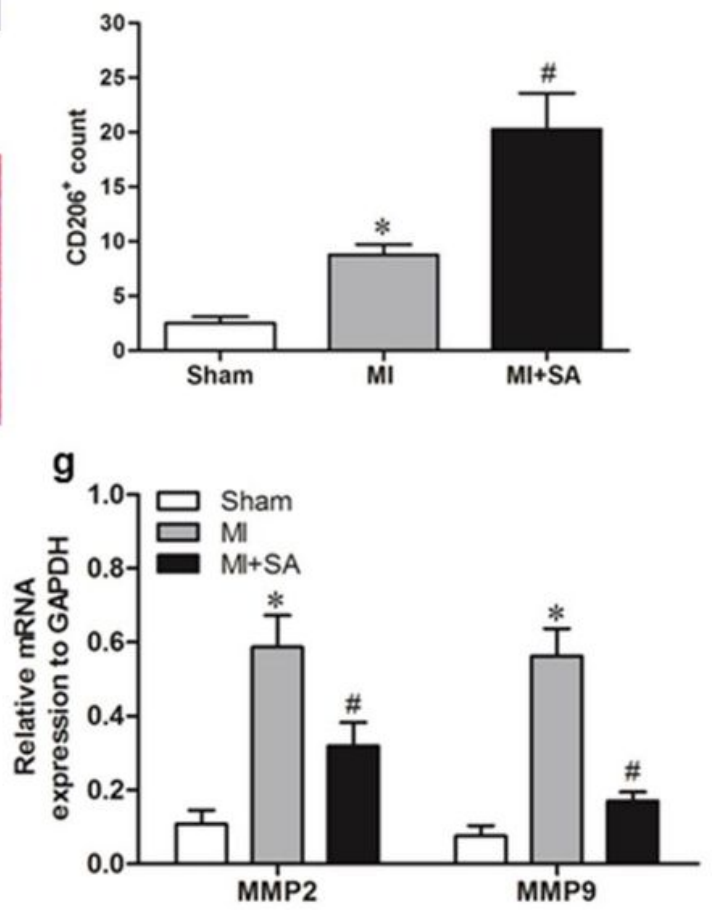

\section{Figure 2}

SA modulated macrophages infiltration at 1 week after $\mathrm{Ml}$ and attenuated myocardial fibrosis at 4 week after MI. a The general macrophage marker CD68 and M2 macrophage marker CD206 were detected by immunostaining analysis. Positive staining of CD68 and CD206 is red. The nuclei were counterstained with DAPI (blue). Scale bar, $100 \mu \mathrm{m}$. b and $\mathbf{c}$ Immunostaining quantitative analysis showed that CD68 expression was decreased and CD206 expression was increased after SA treatment. $\mathbf{d}$ Interstitial fibrosis (blue) detected by masson staining in the sham group, MI group, and Ml+SA group. Scale bar, $50 \mu \mathrm{m}$. e Comparison of collagen volume fraction (CVF). $\mathbf{f}$ and $\mathbf{g}$ Expression of MMP9 and MMP2 proteins in the three groups. *compared with the sham group, $\mathrm{P}<0.05$, \#compared with the Ml+SA group, $\mathrm{P}<0.05$ 

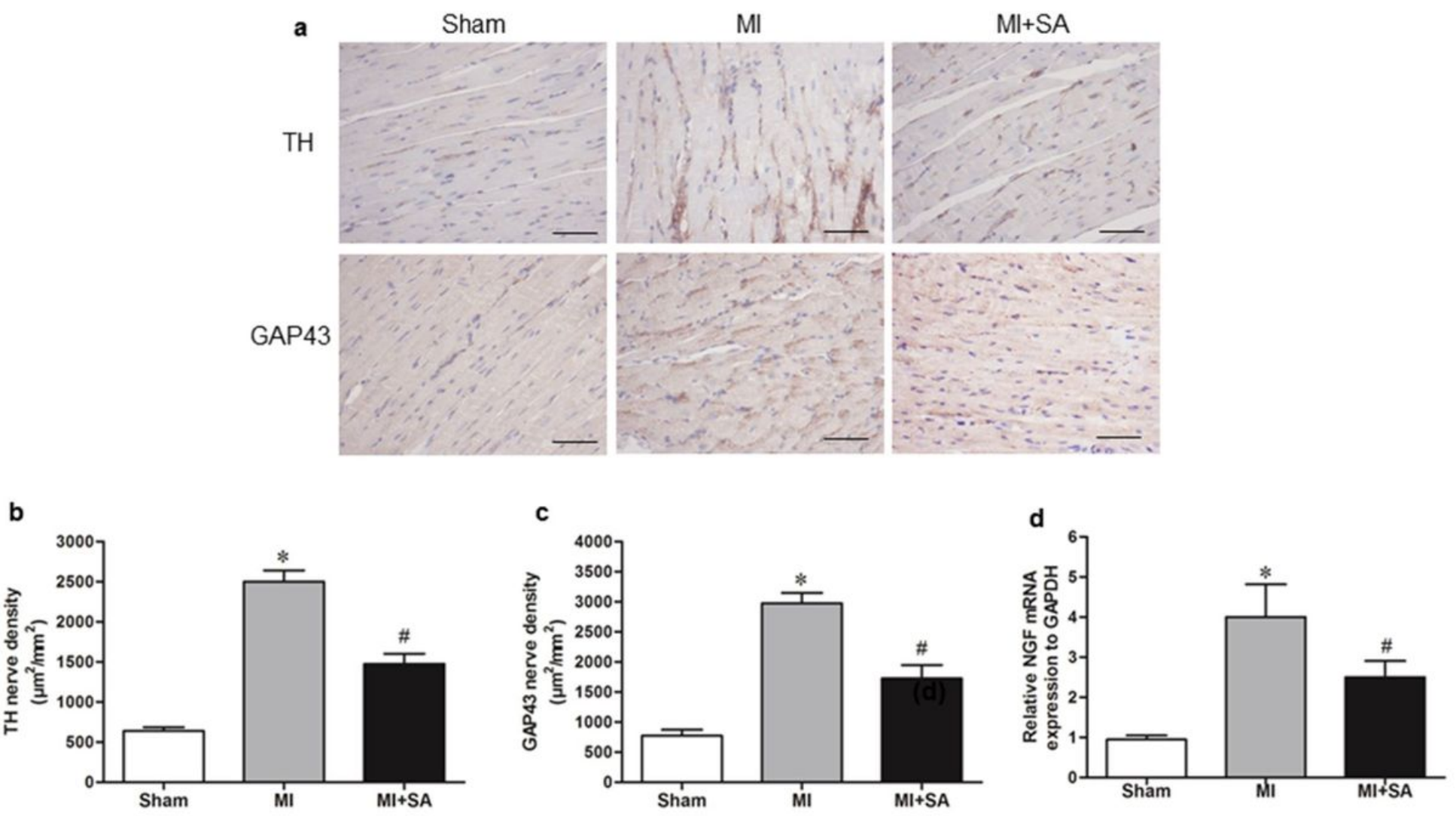

Figure 3

SA attenuated sympathetic nerve remodeling and nerve sprouting at 4 week after MI. a TH and GAP43 were detected by immunohistochemistry. Scale bar, $50 \mu \mathrm{m}$. b and c Quantitative analysis of TH and GAP43. $\mathbf{d}$ Expression of NGF mRNA in the three groups. *compared with the sham group, $\mathrm{P}<0.05$, \#compared with the Ml+SA group, $\mathrm{P}<0.05$ 

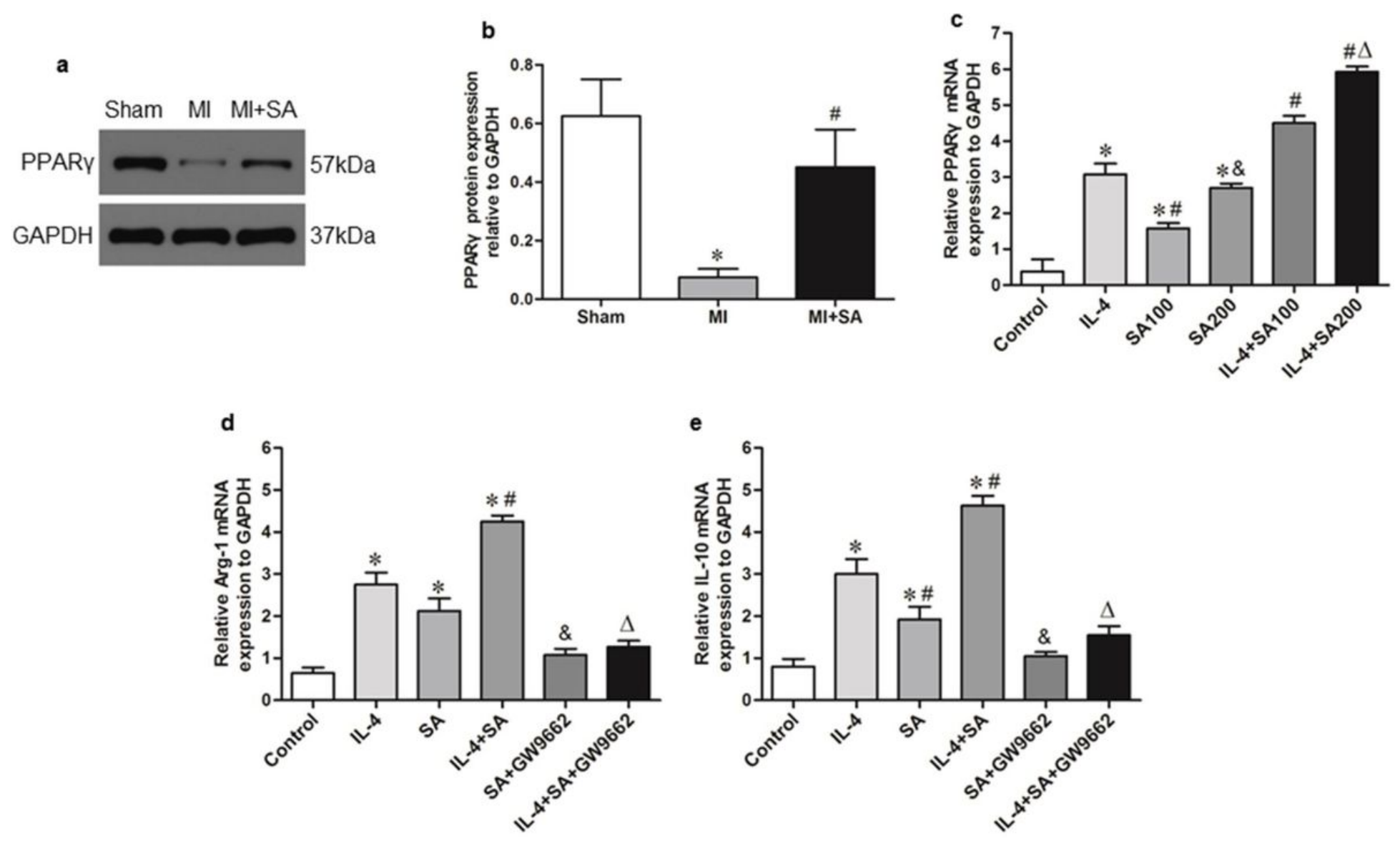

Figure 4

SA activated expression of PPARy and the effect of SA on BMDMs. $\mathbf{a}$ and $\mathbf{b}$ PPARy protein expression was detected by Western blot analysis. *compared with the sham group, $\mathrm{P}<0.05$, \#compared with the Ml+SA group, $P<0.05$. c PPARy mRNA expression in BMDMs and IL-4 induced BMDMs after SA treatment. *compared with the control group, $\mathrm{P}<0.05$, \#compared with the IL-4 group, $\mathrm{P}<0.05$, \&compared with the SA100 group, $\mathrm{P}<0.05 ; \Delta$ compared with the IL-4+SA100 group, $\mathrm{P}<0.05$. $\mathbf{d}$ and $\mathbf{e}$ The effect of SA on Arg1 and IL-10 mRNA expressions in BMDMs and IL-4 induced BMDMs and the effect of PPARY antagonist GW9662 on SA induced Arg1 and IL-10 expressions. *compared with the control group, $P<0.05$, \#compared with the IL-4 group, $P<0.05$, \&compared with the SA group, $P<0.05 ; \Delta$ compared with the IL-4+SA group, $\mathrm{P}<0.05$ 